\title{
ANTARES-IceCube combined search for neutrino point- sources in the Southern Hemisphere
}

\author{
Giulia Illuminati ${ }^{1, *}$ on behalf of the ANTARES and IceCube Collaborations \\ ${ }^{1}$ IFIC - Instituto de Física Corpuscular (CSIC - Universitat de València) c/ Catedrático José Beltrán, 2 \\ E-46980 Paterna, Valencia, Spain
}

\begin{abstract}
A search for cosmic neutrino point-like sources covering the Southern hemisphere is presented, using 9 years and 7 years of data from the ANTARES and IceCube neutrino telescopes, respectively. The advantageous field of view of ANTARES as well as the high statistics provided by IceCube are exploited to open a window in the Southern sky where the sensitivity to point-sources improves by a factor $\sim 2$ compared to individual analyses. An unbinned maximum likelihood method is used to search for a localised excess of muon events over the expected background. Before applying the method to the unblinded dataset, the sensitivity and discovery potential of the search are computed through dedicated pseudo-experiments.
\end{abstract}

\section{Introduction}

Neutrino telescopes can play a crucial role in investigating the physical properties of astrophysical high-energy emitters or in the possible discovery of new astrophysical sources. The high-energy field is currently led by the IceCube [1] and ANTARES [2] experiments. The different characteristics of the two telescopes, in particular the larger instrumented volume of IceCube and the better visibility of the Southern sky for neutrino energies below $100 \mathrm{TeV}$ of ANTARES, allow for a gain in sensitivity by combining the datasets from both experiments in a joint search for point-sources in the Southern Hemisphere. The data samples employed in this search are described in Section 2. The analysis method is presented in Section 3, while in Section 4 the expected performance of the search is shown. The conclusions are given in Section 5 .

\section{Data sample}

The data sample used in this analysis corresponds to all events from the Southern sky which were included in the latest published ANTARES point-source analysis [3], combined with the through-going track-like events used in the seven-year IceCube point-source search [4]. The ANTARES sample corresponds to track-like and shower-like events recorded between early 2007 and the end of 2015. The IceCube samples were recorded from 2008 to 2015 , with the detector operating either in partial (samples IC40, IC59, IC79) or in full (samples IC86, 2012-2015) configuration. A summary of the datasets is given in Table 1.

\footnotetext{
*e-mail: giulia.illuminati@ific.uv.es
} 
Table 1. Characteristic features of the ANTARES and IceCube samples employed in this search. Only Southern-sky events (numbers indicated by last column) have been selected for the present analysis.

\begin{tabular}{ccc}
\hline \hline ANTARES sample & Livetime [days] & \# of events \\
\hline Tracks & 2415 & 5807 \\
Showers & 2415 & 102 \\
\hline \hline IceCube sample & Livetime [days] & \# of events \\
\hline IC40 & 376 & 22779 \\
IC59 & 348 & 64257 \\
IC79 & 316 & 44771 \\
IC86 & 333 & 74931 \\
2012-2015 & 1058 & 119231 \\
\hline
\end{tabular}

Due to the different layouts and locations of the telescopes, each sample has a different efficiency for detecting events from potential sources. The larger size of IceCube guarantees a larger effective area for neutrino energies above $\sim 100 \mathrm{TeV}$. However, in order to minimise the down-going background of atmospheric muons in the sample of through-going tracklike events when looking at the Southern sky, the IceCube point-source analysis introduced a declination-dependent energy cut which strongly suppresses low energy events in the final data sample. ANTARES, which can use the Earth as a filter against atmospheric muons in the Southern sky, maintains a larger effective area in this energy and declination range. The relative contribution of each sample $j, C^{j}(\delta, d \Phi / d E)$, defined as the ratio of the expected number of signal events for the given sample to that for all samples, depends on the source spectrum, $d \Phi / d E$, and declination, $\delta$. Figure 1 shows the relative fraction of signal events for an unbroken $E^{-\gamma}$ spectrum, with $\gamma=2$ (left) and $\gamma=2.5$ (right) as a function of the source declination. While for an $E^{-2}$ spectrum all samples significantly contribute to most of the Southern sky, for a softer spectrum, the contribution of high energy neutrinos is lower, and therefore the relative contribution of the ANTARES sample increases.
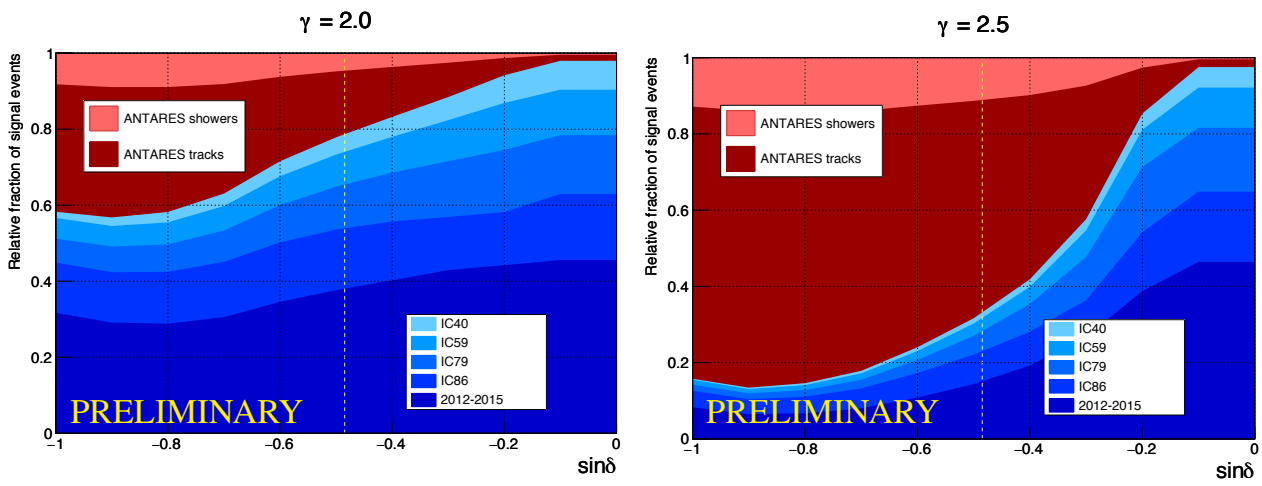

Figure 1. Relative fraction of signal events from each sample as a function of the source declination for an unbroken $E^{-\gamma}$ spectrum, with $\gamma=2$ (left) and $\gamma=2.5$ (right). The contribution of the ANTARES (IceCube) samples is represented by different shades of red (blue). The vertical dashed line marks the declination of the Galactic Centre. 


\section{Analysis method}

A maximum likelihood estimation is performed to identify clusters of cosmic neutrinos from point-like sources over the background of randomly distributed atmospheric neutrinos. The likelihood exploits the energy and directional information of each event to distinguish signallike clusters from clusters produced by random agglomerations of background events. The used likelihood is defined as

$$
L=\prod_{j} \prod_{i \in j}\left[\frac{n_{s}^{j}}{N^{j}} S_{i}^{j}+\left(1-\frac{n_{s}^{j}}{N^{j}} B_{i}^{j}\right)\right],
$$

where $j$ indicates one of the seven data samples (ANTARES tracks, ANTARES showers, IC40, IC59, IC79, IC86 or 2012-2015), $i$ indicates an event belonging to the $j$-th sample, $S_{i}^{j}$ is the value of the signal probability density function (PDF) for the $i$-th event in the $j$-th sample, $B_{i}^{j}$ indicates the value of the background PDF, $N^{j}$ is the total number of events in the $j$-th sample, and $n_{s}^{j}$ is the number of signal events fitted in the $j$-th sample. The number of fitted signal events $n_{s}^{j}$ in each sample is related to the total fitted number of signal events $n_{s}$ by the relative contribution of each sample (introduced in Section 2$), C^{j}(\delta, d \Phi / d E)$, through the relation $n_{s}^{j}=n_{s} \cdot C^{j}(\delta, d \Phi / d E)$.

The signal and background PDFs are given by the product of a directional and an energy term. The definition of these PDFs is slightly different for the ANTARES and the IceCube samples and can be found in [3] and [4], respectively.

In the likelihood maximisation, the values of the number of signal events $n_{s}$ and the signal spectral index $\gamma$ are left free to vary. Values between 1 and 4 are allowed for the spectral index. In order to estimate the signal-likeness of a cluster and to determine the significance of any observation, a test statistic is computed as

$$
Q=\log L\left(\hat{n}_{s}, \hat{\gamma}\right)-\log L\left(n_{s}=0\right),
$$

where $\hat{n_{s}}$ and $\hat{\gamma}$ are the values that maximise the likelihood.

The behavior of $Q$ for different signal strengths is determined from pseudo-experiments (PEs). In each PE, a skymap is generated with a number of background events equal to the number of selected events in data. The declination distribution is drawn from the measured one, while the right ascension distribution is assumed to be uniform. In addition, signal events are injected according to the investigated spectrum at a given source position.

\section{Expected performances}

In order to estimate the potential of the combined search to discover a neutrino source, the $5 \sigma$ discovery flux and the $90 \%$ C.L. sensitivity are calculated for an $E^{-\gamma}$ neutrino spectrum, with $\gamma$ equal to 2.0 and 2.5. The results are shown in Fig. 2 in comparison to the curves from the individual IceCube and ANTARES analyses.

It is found that the sensitivity and the discovery flux improve by a factor $\sim 2$ in different regions of the Southern sky, depending on the energy spectrum of the source. For an $E^{-2}$ spectrum, the most relevant improvement is achieved in a region of the sky that is centred approximately at the declination of the Galactic Centre $\left(\delta=-29^{\circ}\right)$. 

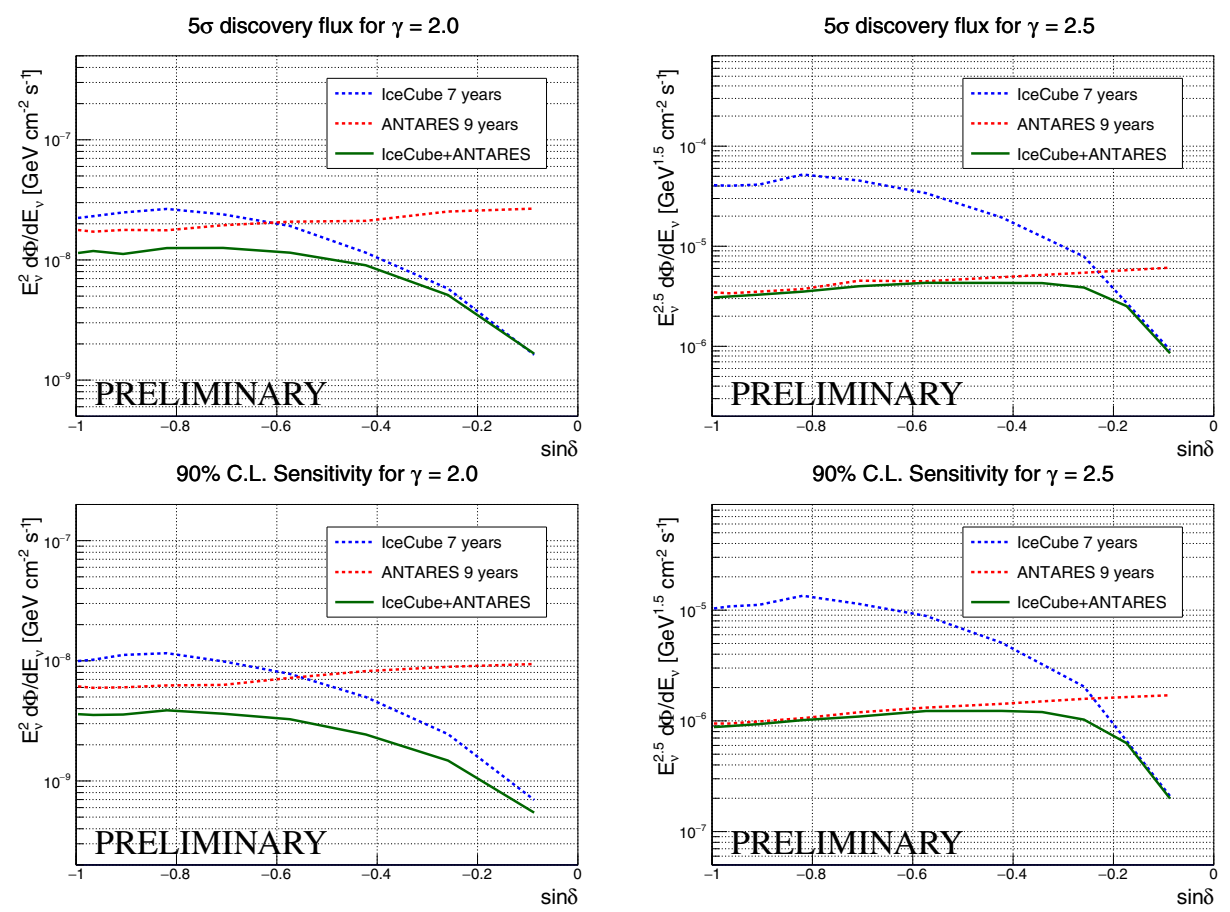

Figure 2. Point-source $5 \sigma$ discovery fluxes (upper plots) and median sensitivities (bottom plots) for an unbroken $E^{-\gamma}$ neutrino spectrum, with $\gamma=2$ (left) and $\gamma=2.5$ (right). The green line indicates the results for the combined search. Blue and red curves show the results for the individual IceCube and ANTARES analyses, respectively.

\section{Conclusions}

A combined point-source analysis of data from the ANTARES and IceCube detectors in the Southern sky has been presented. Preliminary results show that combining the data from both experiments allows to achieve the best point-source sensitivity in critical overlap regions in the Southern sky, where in particular neutrino emission from Galactic sources may be found. This analysis is ongoing, but unblinding of the data is expected in (early) 2019.

We gratefully acknowledge the financial support of Ministerio de Economía y Competitividad (MINECO): Plan Estatal de Investigación (ref. FPA2015- 65150-C3-1-P), (MINECO/FEDER)), Severo Ochoa Centre of Excellence (MINECO), and Prometeo and Grisolía programs (Generalitat Valenciana), Spain.

\section{References}

[1] A. Achterberg et al. (IceCube Collaboration), Astropart.Phys. 26, 155-173 (2006)

[2] M. Ageron et al., (ANTARES Collaboration), Nucl.Instrum.Meth. A656, 11-38 (2011)

[3] A. Albert et al. (ANTARES Collaboration), Phys.Rev. D96 no.8, 082001 (2017)

[4] M. G. Aartsen et al. (IceCube Collaboration), Astrophys.J. 835 no.2, 151 (2017) 